\title{
Importance of vision, visibility and viability of Microbial Resource Centres
}

\author{
Om Prakash*, Avinash Sharma and Yogesh S. Shouche* \\ National Centre for Microbial Resource, National Centre for Cell Science, Pune 411007 India
}

\begin{abstract}
Microbes are providers of raw materials for green, red, blue and white or grey biotechnology. Recent data from DNA-based culture-independent research revealed that huge microbial diversity of immense biotechnological potential is not yet cultured. Cultivation, characterization and biobanking of microbial resources are imperative for academic as well as industrial growth of any nation. Establishment of a quality Microbial Resource Centre (MRC) with state-of-theart facilities and competent faculties is a prerequisite to safeguard microbial biodiversity for future bioeconomy. This article discusses how the vision and visibility of MRCs will help in their long-term sustainability and revenue generation respectively, for the benefit of the research community.
\end{abstract}

Keywords: Biodiversity, bioeconomy, microbes, Microbial Resource Centre.

\section{Microbes are imperative for life}

MiCROBES are tiny, omnipresent and omnipotent components of nature that are non-visible to the naked eye. They operate most of the biogeochemical cycling of materials and produce up to $50 \%$ breathable oxygen ${ }^{1}$. Microbiota is a more recent terminology and is used to denote or represent a complete microbial population of a particular habitat. It includes bacteria, archaea, microalgae, fungi, protozoa and viruses. They are the main pillars of the red, green, white and blue biotechnology ${ }^{1-4}$. In a nutshell, microbes are involved in various activities related to global climate change, energy generation, production of biomolecules for pharmaceuticals and other industries, plant nutrition and soil health ${ }^{5-8}$. On the other hand, they are a good source of enzymes, vitamins and amino acids, and also help in the production of food, fuel and fodder ${ }^{9-13}$. For example, different genera and species of plant growth-promoting rhizobacteria (PGPR) are used as bioinoculants to promote plant health and disease resistance capacity. Application of microbes-based formulations helps to reduce toxic chemicals and promote the concept of sustainability ${ }^{14}$. Methanogenic archaea are responsible for global methane generation and production

*For correspondence. (e-mail: prakas1974@gmail.com; yogesh@nccs.res.in) of biogas, while methanotrophs consume the generated methane and mitigate the greenhouse effects caused by it. Halophilic archaea are producers of valuable chemicals and enzymes of biotechnological importance. Denitrifiers and anoxic ammonia oxidizers are used for efficient nitrogen removal from wastewater-treatment plants ${ }^{15,16}$. Bacteria residing in the human-gut are considered as essential components of human health and diseases ${ }^{17,18}$. The status of animal and human health is directly related to the interactions of pathogens with the host. Thus cultivation, characterization and biobanking of the cultivated organisms without a change in phenotype and functionality are the most important aspects of microbiological research $^{2}$. Recently, culture-independent metagenomic approach has discovered the black box of hidden microbial diversity and unveiled the fact that huge microbial diversity of biotechnological and environmental potential is not-yet cultured ${ }^{2}$. Cultivation of such microbial diversity using recent approaches of culturomics, or directly exploiting their potential without culturing them can open new avenues in different areas of biotechnology ${ }^{19-23}$.

It has been proven that the culture-independent metagenomics approach can only provide information about microbial community structure but not about their functionality and ecological roles ${ }^{2,24}$. Isolated pure culture is considered as one of the best model systems for the study of functionality, ecological role and physiology of microbes. Therefore, interest in cultivation, purification, characterization and preservation of microbes without losing their functionality is once again gaining importance and attention ${ }^{2,7}$. New approaches of cultivation are being implemented ${ }^{9,21,23-26}$. Previously, microbial cultivation, characterization and preservation were considered as the job of a pure taxonomist and no serious microbiologist showed any inclination towards them. Considering the importance of cultivation in taxonomy and other related areas, culturomics is now emerging as an important branch of 'OMICS' ${ }^{24}$. The aim of culturomics is to cultivate and characterize the not-yet-cultured organisms using high-throughput approaches of cultivation and characterization $^{24}$. Unlike traditional cultivation, culturomics uses a wide range of culture conditions and highthroughput identification methods like matrix-assisted laser desorption time-of-flight mass spectrometry (MALDI-TOF/MS) for this purpose ${ }^{27}$. Extensive efforts are in progress to explore the hidden but not-yet-cultured 
microbial diversity and study in the laboratory. Several protocols, new media and novel approaches have been devised to culture these microbes in the laboratory, but there is an urgent need to develop a more systematic and logical approach in this area ${ }^{9,21,23-26}$.

\section{Microbial Resource Centres and their role in diversity conservation}

It is clear from the above discussion that microbes are underpinning biotechnology research and are the backbone of biotech industries for the development of many products and processes. Now, the following question arises: 'how do we explore and exploit the available microbial resources to contribute to sustainable growth and economy of a nation? ${ }^{4,13,28,29}$. To increase the contribution of microbial bioresources in the world's bioeconomy, their cultivation, taxonomical characterization and biobanking are imperative. Establishment of quality Microbial Resource Centres (MRCs) is important for concept $^{30,31}$. MRCs are the real wealth of any nation and almost every country has established its own MRCs or Microbial Repositories to safeguard, authenticate and distribute microbial diversity for research, teaching and industrial exploitation. In addition, MRCs also provide microbial identification services to non-taxonomists for taxonomical identification of their organisms belonging to different biosafety levels (BSLs). They also provide training and standard operating procedures (SOPs) to the users and research community for proper handling of different groups of microbes to prevent the risk of laboratory and environmental contamination. In addition, MRCs should also prioritize cultivation, purification, characterization and inventorization of untapped but valuable organisms of clinical, environmental, agricultural and industrial importance, and develop methods to explore them to boost the bio-based economy of a country ${ }^{32-34}$. Therefore, any MRC should not limit itself only to services, as this restricts the level of technical expertise and hampers its vision for multidimensional expansion. Quality research should be a key component of MRCs to keep pace with the advancement in the field of microbial taxonomy, ecology and genomics for quality services ${ }^{2,35}$. Therefore, a Research and Development Wing in the area of microbial taxonomy, ecology, omics, microbial-related intellectual property rights should be established in all the MRCs to strengthen their in-house activities. Development of innovative protocols, especially in the cultivation of not-yet-cultured organisms, detection of hidden microbes from diverse niches, intact habitat, host and microbiome preservation and strain levels authentication of the microbes is necessary to sustain and maintain the MRCs for longer duration. Establishment of a quality Resource Centre with its entire component for research, reference and training, needs excellent infrastructure and invest- ment ${ }^{13,34,36-38}$. MRCs deal with different groups of microorganisms which need a team of scientists and technical staff with expertise in cutting-edge areas of microbiology for accession, long-term preservation, revival, authentication, validation and distribution of microbial resources to academia and industry based on demand. Due to huge microbial diversity, separate teams of microbiologists with expertise in handling different groups are essential for the expansion and development of a world-class microbial biobank facility (Figure 1). A good MRC should be equipped with: (i) Research and Development Wing; (ii) Service Department for advertising, packaging and supply of bioresources using international norms; (iii) a team of scientists and technical staff for deposition, revival and authentication of the preserved resources; (iv) International Depository Authority (IDA) for depositing cultures for patent purpose and safe deposit facility of customer's resources on payment basis (Figure 1). It should use well-defined protocols and SOPs for accession and benefit-sharing and regular upgradation and ISO certification of the processes ${ }^{39}$. Figure 1 shows the essential wings of an MRC.

\section{Vitality and sustainability of MRCs}

Long-term sustainability of an organization depends on its revenue streams, funding status, mandate for research

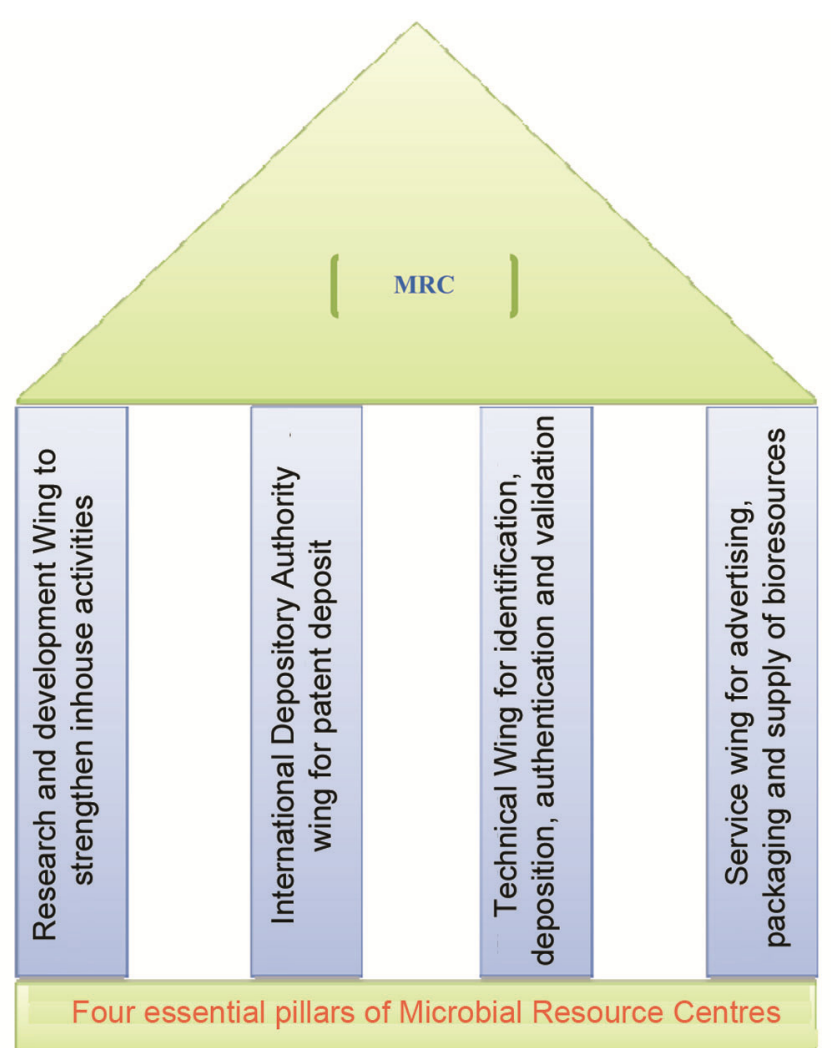

Figure 1. Essential pillars of MRCs for their expansion and growth. 
and societal benefits, and its specific goals and vision ${ }^{38}$. Despite the enormous contribution of MRCs in basic and applied research, they are being ignored by the funding agencies since a long time. It is unlikely for MRCs to sustain on their own revenue due to high cost of preservation, authentication and validation of resources, and the sale of authentic and validated resources at subsidized cost to the academic/research community. Evaluation of the revenue streams of most of the MRCs of the world indicates that they sustain on mixed funding ${ }^{13,34,40}$. To some extent, the revenue is generated by the sale of resources, training, services with major support from government or public funding to the MRCs (Figure 2). Therefore, financial support from the government is imperative for the expansion and sustainability of $\mathrm{MRCs}^{30,33,40}$. The functionality and long-term sustainability of MRCs are based on several other factors like: (i) good infrastructure equipped with cutting-edge research facilities, (ii) potential to attract good faculty and staff trained in the field of taxonomy, biodiversity, cultivation, biobanking and microbial ecology, (iii) potential and expertise for networking with academia and industries, (iv) mandate and capacity for excellence in high standard service facility for customer satisfaction, and (v) financial support for future expansion and long-term sustainability. Therefore, MRCs should follow the concept of farsightedness and attract, develop and train their manpower accordingly ${ }^{31}$. In addition, they should create diversity in terms of their holding and attract expert scientists and staff to foster the anaerobes, archaea, cyanobacteria, fungi, yeast and diverse groups of bacteria under a single umbrella. Thus

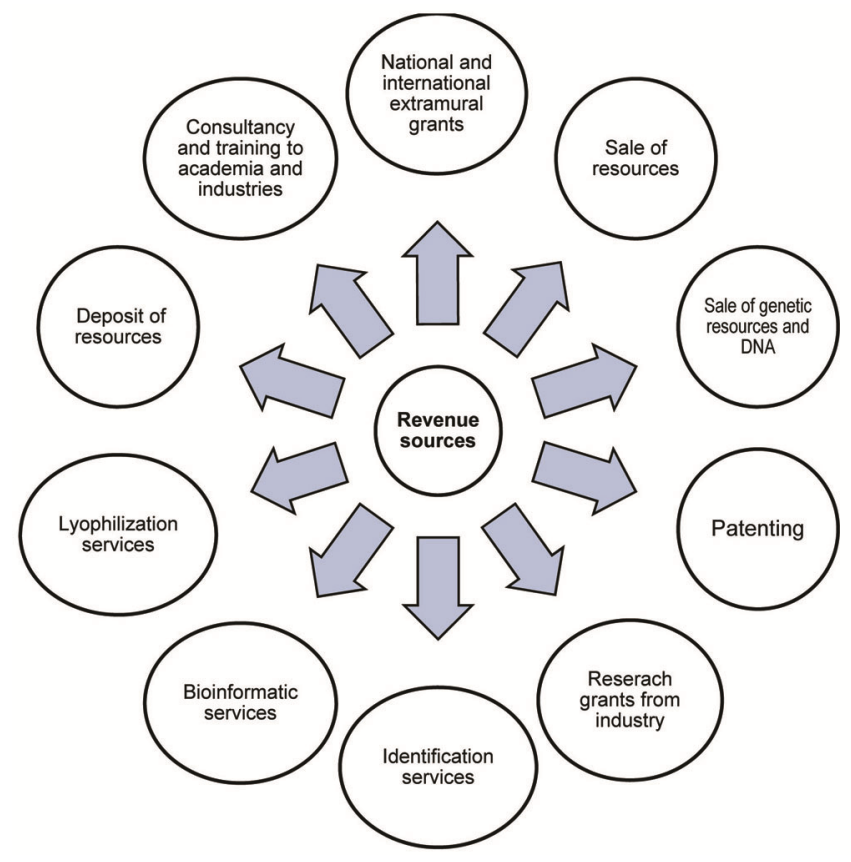

Figure 2. Representation of different sources of revenue of Microbial Resource Centres (MRCs). for long-term sustainability, MRCs should balance their research as well as service activities, and they must be promoted and guaranteed by the funding agencies for expansion of their activities. The funding agencies should understand the importance of preservation of microbial diversity which can be exploited for bioprospecting and economic growth of any nation. A number of microbial products like cheese, tofu, proteases, lipases, etc. are made available in the market for human use due to availability of their standard non-mutated and non-contaminated culture in MRCs. On the other hand, identification and characterization of many pathogens of plant, animal and human origin become possible because their reference strains were available in public collection for comparative study. Furthermore, culture collection is also important because it provides standard strains to study the changes in the genotype of new emerging pathogens, which helps in their treatment and control.

\section{Cost-benefit analysis and investment criteria for MRCs}

It is obvious that for establishing a decent infrastructure and maintaining a team of experts, reasonable amount of budget is needed ${ }^{33,36,37}$. Cost-benefit analysis of MRCs without underestimation of cost and overestimation of profit indicates that benefit always outcompetes the cost of investment. In addition to direct benefits, MRCs also contribute indirectly in terms of generation of trained human resources, and maintenance of microbial diversity to boost the current and future bioeconomy of a nation $^{28,34,37}$. For any nation with major unexplored biodiversity hotspots, the relevance of such organizations is immense in terms of accessioning, characterization, biobanking, authentication and distribution of authentic microbial cultures to academia and industries for biotechnological research. According to some studies, in developing nations where the cost of manpower is 50$60 \%$ cheaper than that in developed nations, the estimated cost of cultivation, characterization and preservation of a single microbial strain is about $€ 400-500$ (refs 34, 40). For a simple calculation, if granting agencies grant 100 projects per annum in microbial diversity and each project cultivates just 10 new strains of bacteria/fungi/ archaea per financial year, then scientists involved in biodiversity research are able to cultivate and characterize 1000 strains in a financial year with a worth of $€ 500,000$. If researchers discard the strains after completion of their projects, then the nations lose their investments as well as important microbial diversity of biotechnological importance. Furthermore, biobanking of such diversity not only preserves the incurred cost on their cultivation, but also uses them for future biotechnological exploitation to boost the bio-based economy of the nation and make raw materials available for academia and industries. 
As mentioned earlier, MRCs generate some part of their revenue through the sale of bioresources, services, consultancy and extramural projects (direct benefit). In addition, they also save a huge amount of national revenue by providing the bioresources and identification services at a subsidized cost to researchers and industries, and prevent the outflow of revenue on their purchase from expensive international markets ${ }^{13,34,40}$. For example, MRCs of developing nations are providing identification services and also selling their quality bio-resources to their academicians, researchers and industries at a rate 56 time cheaper than European and American markets, thus providing direct benefit to their nations and also saving huge costs. In addition to generation of direct revenues by the sale of resources and providing services, there are several indirect benefits of MRCs to any country. For example, they can conduct applied research, develop protocols and standards for biosafety and biosecurity, thus saving the cost on purchase of bioresources from outside for research, training and industries. Thus, if we do the cost-benefit analysis of an MRC of any country, including its direct and indirect contributions, we find that benefit is several folds higher than the incurred cost on its establishment and operation.

\section{National Centre for Microbial Resources: an emerging MRC of India}

The National Centre for Microbial Resource (NCMR) has been established by the Department of Biotechnology, Government of India at National Centre for Cell Science (NCCS), Pune. NCMR is India's evolving culture collection with all the facilities required to fulfil the criteria of a good $\mathrm{MRC}^{35}$. It has been successful for more than 8 years in maintaining and characterizing microbial resources generated during the Microbial Mission Programme of India. The Centre has a well-trained research and technical team of microbiologists with good experience in different areas, including cultivation, preservation, biosafety, metagenomics, genomics, proteomics, intact microbiome preservation and handling capacity of different groups of organisms, including anaerobes, photoautotrophs, fungi, archaea, cyanobacteria, etc. NCMR also provides deposit services like safe, general and patent deposits. The Centre received recognition as an International Depository Authority (IDA) by the World Intellectual Property Organization (WIPO), Geneva, Switzerland, which functions under the norms of the Budapest Treaty ${ }^{41}$, and is continuously improving its functionality, database and protocols.

The goal of NCMR is to promote long-term sustainability in different areas, including agriculture, energy and environment, climate change and medicine. Cultivation, characterization and preservation of important groups of microorganisms and supplying them for research and to the industries is the main aim of the Centre. For example, cultivation, characterization and preservation of PGPR and their supply to industries on collaboration or purchase basis for development of bioinoculants, will promote the concept of sustainable agriculture and reduce the use of chemical pesticides that are harmful to humans as well as the environment. Similarly, cultivation and preservation of pollutant-degrading bacteria and their supply for environmental clean-up will strengthen the concept of environmental sustainability. In a nutshell, the aim of the Centre is cultivation and accession of microorganisms of environmental, agricultural, medicinal and biotechnological importance, and to exploit them with the help of industries and other stakeholders for sustainable development in India.

Although most of the organisms are harmless, a few of them are pathogenic. NCMR has its own inherent policy to provide training regarding good laboratory practices and BSLs to students, trainees and its staff before the start of any work. In addition to lab safety, it also mitigates the potential risk of transmission, contamination and spread of pathogens in the environment. NCMR is also actively involved in the supply of microorganisms to national and international research communities, industries and academic institutions. Therefore, it follows the international norms for depositing, authentication, packaging and transportation of cultures as proposed for MRCs by the Cartagena Protocol on Biosafety (http://www.biodiv.org/biosafety/protocol.asp), Budapest Treaty on the International Recognition of the Deposit of Micro-organisms for the Purposes of Patent Procedure (Bussas et al.; http://www.cnpat.com/worldlaw/treaty/ budapest_en.htm), Convention on Biological Diversity (http://www.biodiv.org/convention/articles.asp), EC Directive 93/88/EEC on Biological Agents (http://europ.eu.int/opnews/395/en/r3633.html) for purchase through Celex EC Directive 90/679/EEC setting mandatory control measures for laboratories http://eur-op.eu.int/ opnews/395/en/r3633) and IATA Dangerous Goods Regulations (http://www.iata.org/cargo/dg/dgr.htm). Along with its expert team of microbiologists, NCMR is striving to fill the gap in the area of microbial ecology, diversity, taxonomy and bioinformatics in India. The Centre is expanding to support all the different activities for services, biobanking and research related to microbial diversity and taxonomy. It is continuously striving to establish new facilities and services. It will also establish a unique repository of microorganisms with extended antimicrobial resistance traits, and parallel work on the generation of Indian standard strains for research related to development of antibiotic resistance in pathogens. NCMR is also aiming at the establishment of animal and human cell line repository for patent processed under the Budapest Treaty, WIPO.

Data on publication, services offered, deposition, etc. indicate that NCMR is performing well in terms of 
Table 1. Current holdings and services offered at National Centre for Microbial Resource

\begin{tabular}{lc}
\hline Categories & Holdings/services \\
\hline Isolates from microbial mission project & 136,499 \\
General deposit & 5962 \\
Patent deposits (under IDA) & 199 \\
Safe deposits & 50 \\
Cultures received from Piramal Life Science Pvt Ltd (bacteria + fungi) & 28,294 \\
Identified strains by 16S rRNA gene sequencing and MALDI-TOF/MS & $>43,000$ \\
Internal and external services for good quality draft whole genome sequence & $>81$ \\
Services for amplicon-based community metagenomics & $>748$ \\
Recovered viability of preserved strains after passaging (sub-culturing) & $99.9 \%$ \\
\hline
\end{tabular}

output. Till date, the centre has published 261 research papers in journals of national and international repute, including 48 novel taxa of bacteria and fungi of industrial, environmental and clinical relevance. It has also sequenced 150 good-quality draft or complete whole-genome sequences and accessioned 5102 bacteria and fungi under general deposit category from various research groups of India and abroad, and supplied 2210 cultures of bacteria and fungi to industries and academia. In addition, it has provided 112,450 identification services under different categories on paid or collaboration basis for boosting biotech-related research in the country. The IDA section of NCMR has accessioned 217 cultures deposited from various research groups for the filing of patents and also submitted 64 cultures under safe-deposit category (Table 1). More details are available at https://www.nccs.res.in/ index.php/TeamsNCCS/Repositories.

\section{Services offered at NCMR}

Since 2011, NCMR is dedicated to providing high-quality identification services for different groups of microbes, including bacteria, archaea, obligate anaerobes, phytoplasma and fungi to academia and industries. At present NCMR is offering services like genome sequencing, targeted amplicon sequencing, Sanger sequencing, FAME analysis, MALDI-TOF-based identification, Biolog-based physiological and biochemical characterization, and cultivation and deposition of obligate anaerobes. Detail information regarding services and holdings is available at https://www.nccs.res.in/index.php/TeamsNCCS/Repositories. In the last decade, NCMR has supplied more than 2500 authentic cultures to industries and academia for research purpose across the country. Apart from identification services, NCMR accepts cultures for long-term preservation under various categories (Table 1). In addition, it has completed first passaging of preserved isolates with a recovery rate (viability) of $85-90 \%$. Several factors affect viability of cryopreserved cultures and play a crucial role in their recovery during passaging activity. A detailed discussion regarding this is available in Prakash et $a l^{7}$. Also, based on available identification, NCMR is holding strains from more than 250 different genera of immense environmental, clinical and biotechnological potential, which can be explored and exploited further to boost the bio-based economy of India.

\section{Importance of visibility of MRCs}

Visibility or popularity of MRCs among researchers, stakeholders and industries is important from the revenue generation point of view and to attain the status of selfsustainability in terms of funding. Popularity is proportional to revenue in case of MRCs. Popularity and quality attract customers and services which generate more revenue, create less dependency and promote selfsustainability of any organization. Although research publications are one of the best methods of publicity for any of the Centre or Department, regarding their nature of work, there are several other impactful ways to increase visibility among the scientific community and industries involved in microbial-based products and processes. Organizing training, workshops, popular talks and putting-up stalls for the resources, services and activities in national and international meetings and conferences and during exhibitions is a good way of gaining visibility and popularity among the scientific community and to attract services. In addition, social networking sites play an important role in spreading information and gaining visibility. However, MRCs should always maintain good quality of services and research for long-term sustainability.

\section{Conclusion}

Microbes provide a large portion of the ecosystem services and serve as raw materials for biotech research and industries. Due to immense agricultural, medicinal and environmental potential, they are the key components of bio-based economy of any nation. Due to their importance, the entire world is working on the cultivation, characterization and exploitation of microbial diversity to boost bio-based economy. Discovery of not-yet-cultured novel microorganisms and characterizing them for biotechnological potential can be an integral part of future 


\section{RESEARCH ACCOUNT}

bio-based economy of the world. Researchers generally pay more attention to applied fields such as nanotechnology, proteomics, genomics, transcriptomics and metabolomics, and the government also provides funds in these areas but neglects research on biodiversity and microbial resources, which is the real strength of a nation and underpins all biotech-related research. With few exceptions, most of the developing nations have only a moderate expertise and only a few centres of excellence which can accommodate, characterize and preserve the diverse group of microbes for future reference, research and training. The status of research in microbial ecology, diversity and taxonomy in developing nations is not well established and needs more attention. Considering the available biodiversity and microbial resources, establishment of MRCs with state-of-the-art facility is the current need of developing nations.

Furthermore, data on revenue streams of MRCs indicate that they certainly need public and government support for long-term sustainability, development, existence and expansion. Cost-benefit analysis indicates that the benefit of establishing MRCs always outcompetes the incurred cost. In conclusion, investment in MRCs will provide long-term benefits to a nation in terms of sustainable development for human resource and future bioeconomy of the world. MRCs generally work as model systems for the handling of different groups of microbes and provide training to university departments, institutional laboratories, etc. working with microbes. There is no concern, regarding environmental risk, sustainability and feasibility of MRCs if they follow the established norms and SOPs. Generally, MRCs have well-trained scientists and technicians with cutting-edge knowledge of handling different groups of organisms and the knowhow to dispose them. Unlike other areas, interest of students in microbial ecology, diversity and taxonomy is least due to lack of proper funding and quality research in these areas. Therefore, good microbial taxonomists and ecologists those who can take care of our valuable microbial resources in the coming future is the need of the current era. Some developed nations are also collaborating with biodiversity-rich nations to exploit them. Establishment of quality Resource Centres with excellent infrastructure and expertise in biodiversity for future bioeconomy must be part of the intellectual vision of the government and funding agencies of any country. In conclusion, vision and farsightedness are important to make MRCs visible, vital and sustainable for a long duration for service of the nation, science and society.

1. Prakash, O. et al., Polyphasic approach of bacterial classification - an overview of recent advances. Indian J. Microbiol., 2007, 47, 98-108.

2. Prakash, O., Shouche, Y., Jangid, K. and Kostka, J. E., Microbial cultivation and the role of microbial resource centers in the omics era. Appl. Microbiol. Biotechnol., 2013, 97, 51-62.
3. Zipperer, A. et al., Human commensals producing a novel antibiotic impair pathogen colonization. Nature, 2016, 535, 511-516.

4. Ramos, V., Morais, J., Castelo-Branco, R., Pinheiro, Â., Martins, J., Regueiras, A. and Moreira, C., Cyanobacterial diversity held in microbial biological resource centers as a biotechnological asset: the case study of the newly established LEGE culture collection. J. Appl. Phycol., 2018, 30, 1437-1451.

5. Prakash, O. et al., Geobacter daltonii sp. nov., an $\mathrm{Fe}(\mathrm{III})-$ and uranium(VI)-reducing bacterium isolated from the shallow subsurface exposed to mixed heavy metal and hydrocarbon contamination. Int. J. Syst. Evol. Microbiol., 2010, 60, 546-553.

6. Prakash, O. et al., Rhodanobacter denitrificans sp. nov., isolated from nitrate-rich zones of a contaminated aquifer. Int. J. Syst. Evol. Microbiol., 2012, 62, 2457-2462.

7. Prakash, O., Nimonkar, Y. and Shouche, Y. S., Practice and prospects of microbial preservation. FEMS Microbiol. Lett., 2013, 339, $1-9$.

8. Canion, A., Prakash, O., Green, S., Jahnke, L., Kuypers, M. and Kostka, J. E., Isolation and physiological characterization of psychrophilic denitrifying bacteria from permanently cold Arctic fjord sediments (Svalbard, Norway). Environ. Microbiol., 2013, 15, 1606-1618; doi:10.1111/1462-2920.12110.

9. Nichols, D. et al., Use of ichip for high-throughput in situ cultivation of 'uncultivable' microbial species. Appl. Environ. Microbiol., 2010, 76, 2445-2450.

10. Million, M. et al., Increased gut redox and depletion of anaerobic and methanogenic prokaryotes in severe acute malnutrition. Sci. Rep., 2016, 6, 26051.

11. Sharma, R., Prakash, O., Sonawane, M. S., Nimonkar, Y., Golellu, P. and Sharma, R., Diversity and distribution of phenol oxidase producing fungi from Soda Lake and description of Curvularia lonarensis sp.nov. Front. Microbiol., 2016, 7, 1847; doi:org/ 10.3389/fmicb.2016.01847

12. McCluskey, K., Alvarez, A., Bennett, R., Bokati, D., BoundyMills, K., Brown, D. and Dye, G., The US culture collection network lays the foundation for progress in preservation of valuable microbial resources. Phytopathology, 2016, 106, 532-540.

13. Philip, J. and Atlas, R., Microbial resources for global sustainability. In Microbial Resources from Functional Existence in Nature to Applications (ed. Kurtböke, I.), Academic Press, Cambridge, Massachusetts, United States, 2017, pp. 77-101.

14. Keswani, C. et al., Readdressing the biosafety issues of plant growth promoting rhizobacteria. Sci. Total Environ., 2019, 690, 841-852; https://doi.org/10.1016/j.scitotenv.

15. Kuenen, J. G., Anammox bacteria: from discovery to application. Nature Rev. Microbiol., 2008, 6, 320.

16. Nimonkar, Y. S., Yadav, B., Talreja, P. and Prakash, O., Assessment of the role of wastewater treatment plant in spread of antibiotic resistance and bacterial pathogens. Indian J. Microbiol., 2019, 59, 261-265; https://doi.org/10.1007/s12088-019-00793-2e.

17. Prakash, O., Munot, H., Nimonkar, Y., Sharma, M., Kumbhare, S. and Shouche, Y. S., Description of Pelistega indica isolated from human gut. Int. J. Syst. Evol. Microbiol., 2014, 64, 1389-1394; doi:10.1099/ijs.0.059782-0.

18. Prakash, O., Nimonkar, Y., Vaishampayan, A., Mishra, M., Kumbhare, S., Josef, N. and Shouche, Y. S., Description of Pantoea intestinalis sp. nov.: isolated from human gut. Int. J. Syst. Evol. Microbiol., 2015, 65, 3352-3358; doi:10.1099/ijsem.0.000419.

19. Cangelosi, G. A. and Meschke, J. S., Dead or alive: molecular assessment of microbial viability. Appl. Environ. Microbiol., 2014, 80, 5884-5891.

20. La, S. B., Khelaifia, S., Lagier, J. C. and Raoult, D., Aerobic culture of anaerobic bacteria using antioxidants: a preliminary report. Eur. J. Clin. Microbiol. Infect. Dis., 2014, 33, 1781-1783.

21. Browne, H. P. et al., Culturing of 'unculturable' human microbiota reveals novel taxa and extensive sporulation. Nature, 2016, 533, 543-546. 
22. Dione, N., Khelaifia, S., La, S. B., Lagier, J. C. and Raoult, D. A., Quasi-universal medium to break the aerobic/anaerobic bacterial culture dichotomy in clinical microbiology. Clin. Microbiol. Infect., 2016, 22, 53-58.

23. Khelaifia, S., Lagier, J. C., Nkamga, V. D., Guilhot, E., Drancourt, M. and Raoult, D., Aerobic culture of methanogenic archaea without an external source of hydrogen. Eur. J. Clin. Microbiol. Infect. Dis., 2016, 35, 985-991.

24. Lagier, J. C. et al., Culturing the human microbiota and culturomics. Nature Rev. Microbiol., 2018, 16, 540-550.

25. Ma, L. et al., Gene-targeted microfluidic cultivation validated by isolation of a gut bacterium listed in Human Microbiome Project's most wanted taxa. Proc. Natl. Acad. Sci. USA, 2014, 111, 97689773.

26. Tidjani Alou, M. et al., Gut bacteria missing in severe acute malnutrition. Can we identify potential probiotics by culturomics. Front. Microbiol., 2017, 8, 899.

27. Rahi, P., Prakash, O. and Shouche, Y. S., Matrix-assisted laser desorption/ionization time-of-flight mass-spectrometry (MALDITOF MS) based microbial identifications: challenges and scopes for microbial ecologists. Front. Microbiol., 2016, 7, 1359; doi:10.3389/fmicb.2016.01359.

28. Antunes, A., Stackebrandt, E. and Lima. N., Fueling the bio-economy: European culture collections and microbiology education and training. Trends. Microbiol., 2016, 24, 77-79.

29. Sharma, R., Nimonkar, Y., Singh, R., Sharma, A. and Prakash, O., Concept of microbial preservation: past, present and future. In Microbial Resource Conservation, Springer India, 2018, pp. 3554; doi:10.1007/978-3-319-96971-8.

30. Schüngel, M. and Stackebrandt, E., Microbial Resource Research Infrastructure (MIRRI): infrastructure to foster academic research and biotechnological innovation. Biotechnol. J., 2015, 10, 17-19.

31. Van Hop, D., Establishment and management of culture collections of microorganisms (mBRC): an overview. In Microbial Resource Conservation, Springer, Cham, Switzerland, 2018, pp. 55-109.

32. Stackebrandt, E., Schüngel, M., Martin, D. and Smith, D., The microbial resource research infrastructure MIRRI: strength through coordination. Microorganisms, 2015, 3, 890-902.

33. Schüngel, M., Smith, D., Bizet, C., Stackebrandt, E. and the MIRRI Consortium. The role of the European microbial resource research infrastructure project. Enliven: Microb. Microb. Tech., 2014, 1, 001.
34. Overmann, J. and Smith, D., Microbial resource centers contribute to bioprospecting of bacteria and filamentous microfungi. In Bioprospecting, Springer, Cham, Switzerland, 2017, pp. 51-79.

35. Sharma, A. and Shouche, Y., Microbial culture collection (MCC) and International Depositary Authority (IDA) at National Centre for Cell Science, Pune. Indian J. Microbiol., 2014, 54, 129-133.

36. Suzuki, K. I., An overview of Biological Resource Centermaintenance of microbial resources and their management. In Microbial Resources from Functional Existence in Nature to Applications (ed. Kurtböke, I.), Academic Press, 2017, pp. 257 274.

37. McCluskey, K., A review of living collections with special emphasis on sustainability and its impact on research across multiple disciplines. Biopreserv. Biobank., 2017, 15, 20-30.

38. Paterson, R. R. M., Lima, N., Brooksbank, C., Guarini, E., Pasterk, M. and Lavitrano, M., Microbiology managers: managerial training in the RI train project. Trends Microbiol., 2017, 25, 425428.

39. Dedeurwaerdere, T., Broggiato, A. and Manou, D., Global scientific research commons under the Nagoya Protocol. In Common Pools of Genetic Resources: Equity and Innovation in International Biodiversity Law (eds Kamau, E. C. and Winter, G.), Routledge, 2013, p. 224.

40. Smith, D., McCluskey, K. and Stackebrandt, E., Investment into the future of microbial resources: culture collection funding models and BRC business plans for biological resource centres. Springerplus, 2014, 3, 81 .

41. Vera, B., Sharma, A. and Shouche, Y. S., IP and the budapest treaty depositing biological material for patent purposes in microbial resources from functional existence in nature to applications (ed. Kurtböke, I.), Academic Press, Cambridge, Massachusetts, United States, 2017, pp. 275-292.

ACKNOWLEDGEMENTS. This work was supported by the Department of Biotechnology, Government of India, through a grant (Grant no. BT/Coord.II/01/03/2016 dated 6 April 2017), for the establishment of NCMR in Pune. We thank Manali Vaijanapurkar and Dr Tapan Chakrawarti (NCMR-NCCS, Pune) for a critical reading of the manuscript.

Received 14 March 2020; revised accepted 27 May 2020

doi: $10.18520 / \mathrm{cs} / \mathrm{v} 119 / \mathrm{i} 4 / 625-631$ 